\title{
How to perform posterior wall isolation in catheter ablation for atrial fibrillation
}

Hariharan Sugumar MBBS ${ }^{1,2,3,4}$ Stuart P Thomas ${ }^{5}$ MBBS PhD, Sandeep Prabhu MBBS ${ }^{1,2,3,4}$, Aleksandr Voskoboinik MBBS ${ }^{1,2,3,4}$ and Peter M Kistler MBBS PhD ${ }^{1,2,3,4}$

Short title: How to perform PWI

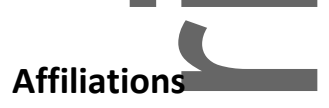

The Baker Heart \& Diabetes Institute, Melbourne, Australia ${ }^{1}$; The Alfred Hospital, Melbourne, Australia ${ }^{2}$; Royal Melbourne Hospital, Melbourne, Australia ${ }^{3}$; University of Melbourne, Melbourne, Australia ${ }^{4}$; Westmead Hospital, University of Sydney and Macquarie University, Sydney, Australia ${ }^{5}$

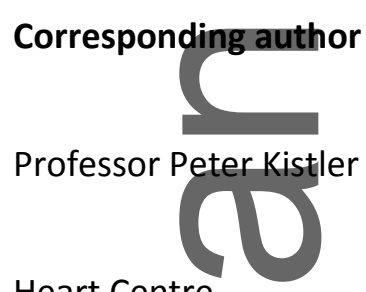

Heart Centre

The Alfred Hospital

Commercial Road, Melbourne, Victoria, Australia 3004

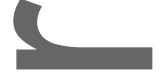

Tel: +61 385321111 , Fax: +61 38532 1111,

Email: peter.kistler@baker.edu.au

Disclosures: None

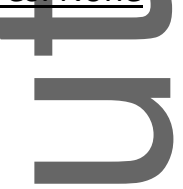

This is the and mascript accepted for publication and has undergone full peer review but has not been thr o t t copyediting, typesetting, pagination and proofreading process, which may lead to differences betwo this version and the Version of Record. Please cite this article as doi:

10.1111/jce.13397.

This article is protected by copyright. All rights reserved. 


\section{Abstract}

Catheter ablation (CA) has become standard of care in patients with symptomatic atrial fibrillation (AF). Although there have been significant advances in our understanding and technology, a substantial proportion of patients have ongoing AF requiring repeat procedures. Pulmonary vein isolation (PVI) is the cornerstone of AF ablation; however, it is less effective in patients with persistent as opposed to paroxysmal atrial fibrillation (PAF). Left atrial posterior wall isolation (PWI) is commonly performed as an adjunct to $\mathrm{PVI}$ in patients with persistent $\mathrm{AF}$ with non-randomized studies showing improved outcomes. Anatomical considerations and detailed outline of the various approaches and techniques to performing PWI are detailed, and advantages and pitfalls to assist the clinical electrophysiologist successfully and safely complete PWI are described.

Keywords: posterior wall isolation, ablation techniques, atrial fibrillation, catheter ablation

\section{Introduction}

Catheter ablation (CA) has become standard of care in patients with symptomatic atrial fibrillation (AF). Although there have been significant advances in our understanding and technology, a substantial proportion of patients have ongoing AF requiring repeat procedures. Pulmonary vein isolation (PVI) is the cornerstone of AF ablation however it is less effective in patients with persistent as opposed to paroxysmal atrial fibrillation (PAF) ${ }^{1}$. Substrate modification beyond the pulmonary veins (PVs) had shown promise in non-randomized studies. However, the landmark STAR AF II trial ${ }^{2}$ demonstrated that linear ablation or targeting of complex fractionated electrogram (CFE) did not improve outcomes beyond $\mathrm{PVI}$ alone. In an attempt to improve outcomes for patients undergoing CA for persistent AF (PSAF), electrophysiologists continue to investigate strategies beyond PVI. This may involve focal impulse and rotor modulation (FIRM) ablation ${ }^{3}$, isolation of regions of low voltage $^{4}$, targeting parasympathetic ganglia, the step-wise approach ${ }^{5}$ or left atrial (LA) posterior wall isolation $(\mathrm{PWI})^{6}$.

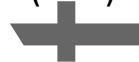

The LA posterior wall (PW) and the PVs share a common embryonic origin ${ }^{7}$. The LA posterior wall (PW) and the PVs share a common embryonic origin. Embryologically, the pulmonary vein incorporates into the smooth walled left atrium and as such shares common histology ${ }^{8}$. There are extensive parasympathetic neural plexi involving the posterior wall extending to the PV antrum, which play an important role in the initiation and maintenance of $\mathrm{AF}^{9}$. 
Posterior wall isolation was not assessed during the STAR AF II trial in PSAF. ${ }^{2}$ Proposed benefits of the addition of PWI to PVI include reinforcement of posterior PV ablation lines or a "belt and braces" approach to PVI: modification of autonomic ganglia, inclusion of proximal triggers or rotors and anatomic debulking of the atrium. Non-randomized studies have suggested improved outcomes in patients with persistent atrial fibrillation ${ }^{10}$. PWI may also be considered in patients with documented recurrent atrial fibrillation despite enduring pulmonary veins isolation.

\section{Left atrial posterior wall: Anatomy \\ Boundaries of the left atrial posterior wall:}

- Superiorly, the PW is bound by the LA roof defined as a cranial line connecting the most superior aspect of the superior pulmonary veins.

- Inferiorly, the most caudal line connecting the most inferior aspect of the inferior pulmonary veins.

- Lateral and medial borders are bounded by the posterior left and right veno-atrial junctions.

The LA PW is a complex structure with significant variation in thickness of the septopulmonary bundle (SBP) (figure 1A), which contributes to majority of the PW. The PW becomes thinner away from the veno-atrial junction. Depending on the vein, thickness ranges between 2 to $4.5 \mathrm{~mm}$ and decreases by about $1.5 \mathrm{~mm}$ at $1 \mathrm{~cm}$ from the junction. ${ }^{11}$ The SBP originates anterior to the interatrial groove behind the anterior interatrial band (Bachmann's bundle) and passes over the roof. The superior margin at the roof is the thickest part of the left atrium measuring up to $6.5 \mathrm{~mm}^{11,12}$. The SBP then descends posteriorly and then inferoseptally between the right and left pulmonary veins. ${ }^{12}$ This explains the relative increase in atrial wall thickness at the right posterior antrum compared with left posterior antrum. As the SBP descends inferiorly, the thickness decreases considerably to $2.3+/-0.9$ $\mathrm{mm}$ between the superior pulmonary veins and decreases further between the inferior pulmonary veins (figure $1 \mathrm{~A})^{13}$. The esophagus and the posterior left atrial wall are adjacent to one another. Typical anatomical relationship is shown in figure 1B.

\section{Prior studies of posterior wall isolation}

Electricalisolation of the PW has an unequivocal electrophysiological endpoint although on occasion challenging to obtain ${ }^{14}$. PWI been shown to be a useful strategy in patients with PsAF compared with PVI alone. A recent meta-analysis that included 594 patients demonstrated a reduction in recurrent AF with PWI ${ }^{10,15-20}$. In addition, a recent randomized study of 120 patients with PsAF demonstrated a $20 \%$ reduction in recurrent AF with PWI. ${ }^{16}$ However, the study was confounded by additional linear ablation involving left anterior, perimitral and cavotricuspid isthmus ablation ${ }^{16}$. Mohanty et al showed improved outcomes with the addition of PWI to PVI. However, this was a three-way randomization targeted at 
assessing focal impulse and rotor modulation (FIRM) ablation. ${ }^{20}$ One hundred and thirteen patients were assessed in a 1:1:1 fashion comparing FIRM only (group 1), FIRM + PVI (group 2) and PVI + PWI and non-PV triggers (group 3). Freedom from AF/AT was highest in group 3(14\% in group 1 vs $52 \%$ in group 2 vs $76 \%$ in group 3 , p-value $<0.0001)$. McLellan et al published the largest series to date of patients with PsAF undergoing posterior wall isolation in addition to PVI. ${ }^{6}$ The multi-procedure freedom from atrial arrhythmias was $85 \%$ at a mean follow-up of $19 \pm 8$ months. The CAMERA-MRI study demonstrated marked recovery of left ventricular function in patients with persistent AF and dilated cardiomyopathy randomized to catheter ablation, which included PVI plus PWI compared with medical rate control. ${ }^{21}$

\section{Techniques for electrical exclusion of the posterior left atrium and pulmonary veins}

The first posterior wall isolation procedures were performed as part of the Cox Maze procedure $^{22}$. This was an adaptation of the incisions used during heart transplantation. Early attempts to reproduce these lesions percutaneously were limited by the available equipment. After improvements in mapping systems and ablation, three effective percutaneous techniques were described. These are wide circumferential pulmonary vein isolation and posterior box ${ }^{10}$, single ring or box isolation of veins and posterior wall together ${ }^{23-25}$ and exclusion of the posterior wall by segmental isolation using the circular mapping catheter ${ }^{18 \text {, }}$ 26 .

Posterior wall isolation may be achieved with a variety of techniques: Wide antral PVI plus posterior box, single ring, "Touching rings" and debulking / extensive posterior wall ablation (figure 2).We aim to provide some "tips and tricks" to achieve electrical isolation and avoid complications. Adetailed outline of the various approaches including advantages and pitfalls to assist the clinical electrophysiologist successfully and safely complete PWI is described.

\section{PWI approach 1: Wide antral pulmonary vein isolation (PVI) plus posterior box.}

This approach involves wide antral PVI followed by inferior and roof lines to achieve posterior wall isolation (figure 2). Contact force enabled irrigated ablation catheters are generally used. A steerable sheath may be used if contact force cannot be adequately achieved. A schematic of the steps involved in this approach is shown in figure 3.

Step 1: Circumferential Pulmonary Vein Isolation (CPVI) involving posterior wall: Pulmonary vein isolation techniques have been described in great detail previously. ${ }^{6,27}$ Generally, for right and leftsided veins, complete contiguous ablation of the anterior segments at $30 \mathrm{~W}$ is completed before targeting the posterior wall. Ablation is limited between 15 and 30seconds and $25 \mathrm{~W}$ on the posterior wall at each site and may be further limited by oesophageal temperature changes. If electrical isolation is achieved before completion of the posterior antral lines then the ablation ring is not completed $^{28}$. HRS guidelines provide a class $2 \mathrm{~A}$ recommendation for the use of an esophageal temperature probe ${ }^{29}$. In our practice, power delivery is interrupted if the temperature increases by more than one degree from baseline. In addition to the actual change or rise in temperature, 
rapidity of change is a marker to immediately terminate ablation at that site and provides sufficient reason to alter the course of the ablation line. It is important to note that ablation over the posterior wall is performed with caution even in the absence of temperature changes as temperature probes vary in electrode number and provide intraluminal rather than adventitial temperature readings ${ }^{29}$. Anteriorly, ablations will be performed up to 60seconds to complete the lesion set in the absence of good local electrogram. Technique of lesion creation is left to the discretion of the operator. In general, we perform lesions as point by point or utilize dragging technique aiming to maintain high continuity index.

The lasso is placed in all four veins upon completion of the circumferential ablation with pacing from the coronary sinus catheter to demonstrate entrance block. Exit block can also be performed at this time depending on operators preference / practice.

\section{Step 2: Inferior or Floor line (figure 3):}

If $\mathrm{AF}$ has been persistent following PVI then direct current cardioversion is performed to restore sinus rhythm. A STAR AF II sub study demonstrated improved outcomes in patients who underwent ablation in sinus rhythm even if this was restored by electrical cardioversion early in the procedure ${ }^{30}$. The procedural endpoint is PVI and PW isolation rather than AF termination with ablation.

The floor line is performed first during pacing from the coronary sinus as conduction block is generally clear given the close proximity of the CS to the inferior line. The floor line is performed by delivering a contiguous ablation line joining the most inferior margin of the inferior PVs at 25W with the circular mapping catheter positioned at all times on the posterior wall. Ablation begins at the left inferior pulmonary vein (LIPV) and is delivered locally until there is electrogram separation or up to 30 seconds moving progressively towards the right inferior pulmonary vein (RIPV) preferring contiguity during the ablation. With gentle clockwise torque on the catheter and the sheath, the catheter sweeps towards the RIPV. Floor line block is confirmed by the presence of widely spaced fixed double potentials and a change in the activation detour from superior to inferior direction on the posterior wall (figure 3). It is critical to achieve inferior line block before embarking on the roof line. Post wall isolation can become most challenging if the roof line is commenced before block is achieved at the floor line as multiple conduction breaks at roof and floor lines makes mapping points of breakthrough difficult.

Step 3: Roof line: 'RIght From LEft (RIFLE)' ablation technique:

Ablation at the roof line is delivered at 25W (direct approach) to $30 \mathrm{~W}$ (RIFLE technique) at the most superior posterior aspect of the LA away from the oesophagus as visualized on preop CT or MRI. The RIFLE technique or RIght From LEft (RIFLE)' approach is an alternative to the direct approach and provides excellent stability. Contact can be titrated generally aiming for a contact force of $15-30 \mathrm{~g}$ at $30 \mathrm{~W}$ for up to 60 seconds or until there is local electrogram separation or attenuation. Retracting the 
catheter into the sheath or retroflexion with a bidirectional catheter increases contact force. Care must be taken to monitor impedance and contact force as a sudden straightening of the catheter can perforate the LA roof.

RIFLE technique: An SL1 sheath is advanced into the left atrium with the ablation catheter about 4-5 $\mathrm{cm}$ out of the sheath and fully $\mathrm{D}$ flexed pointing inferiorly. The sheath and catheter is then advanced towards the left superior pulmonary vein (LSPV) under fluoroscopy (figure 4). The left atrial appendage is avoided as the roof line is more posterior directed and the appendage is vulnerable to perforation. The sheath is then clocked 180degrees pointing the ablation catheter to the right-sided veins. The $\mathrm{D}$ curve can be released to bring the catheter tip in contact with the local tissue near the RSPV. It is important to confirm on fluoroscopy that the catheter remains coaxial to the sheath on fluoroscopy. Gentle movement of the sheath will provide stable catheter control. Clocking and anticlocking the sheath will move the catheter more anteriorly / cranially and posteriorly / caudally, respectively.Pushing the entire assembly towards the LSPV will bring the catheter tip away from the vein into the atrium. (See Table 1). If the catheter falls into the RSPV, it is important to carefully maneuver the catheter out of the vein before withdrawing the assembly under fluoroscopic vision as the catheter can wedge deeper into the vein. A fluoroscopic video of the initial steps involved in RIFLE technique is included in the supplementary section.

Bidirectional block with linear ablation maybe difficult to achieve ${ }^{31}$ particularly at the roof that is the thickest region of the septopulmonary bundle. If electrical connection persists to the posterior wall after completion of roof and inferior lines then, firstly, both lines are mapped with the ablation catheter for fractionated or closely spaced double potentials and ablated. Secondly, if catheter manipulation or contact force has been difficult to achieve, a steerable sheath is taken and a direct approach to the roof line adopted. Thirdly, pacing from the ablation catheter positioned immediately anterior to the roof line is performed with the circular mapping catheter on the posterior wall to assess the integrity of this line. The same approach is then taken to the floor line with pacing immediately posterior, as conduction may recover at the floor line during ablation at the roof. If there are no electrograms along the original lines at the site of earliest activation, then ablation may be performed within the posterior 'box' immediately adjacent to the earliest site.

Finally, if additional ablation adjacent to the original lines is unsuccessful, the earliest activation is directly ablated using the circular mapping catheter akin to the approach taken for PW debulking.

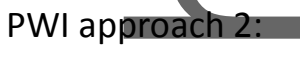

Single ring approach. $^{23,32}$

A single ring of ablation is performed starting at the anterolateral margin of the ridge separating the left-sided veins and the left atrial appendage (figure 2). A deflectable sheath may help to maintain consistent contact force during ablation. Ablation is generally commenced at the inferior margin of the ridge and continues below the left inferior pulmonary veins in a line toward the inferior margin of the right inferior pulmonary vein. Ablation for this line on the posterior left atrial wall is limited to $30 \mathrm{~W}$ with a maximum 
ablation duration of 30s. Ablation duration is shortened depending on esophageal temperature rises, large impedance falls or rapid attenuation of signals. As noted above, pacing from the coronary sinus can be used to identify progressive bipolar signal splitting during ablation. Delayed activation may also be seen in a circular mapping catheter placed in the left veins. Ablation may beperformed at up to $40 \mathrm{~W}$ for a maximum of $30 \mathrm{~s}$ on the anterolateral side of the appendage-left vein ridge. However, if this approach is chosen it is frequently necessary to place additional lesions inside the ridge at the level of the left carina at lower power. After passing around the superior margin of the left superior vein, a roof line is placed from the superior left vein to the superior margin of the right superior vein. It is difficult to complete this line without curving posteriorly between the veins where the muscle is thinner. If this path is chosen we recommend monitoring esophageal temperature opposite the ablation site. The most challenging section of the roof line is at the site of the septopulmonary bundle. In some cases, it is necessary to ablate below the roof line to interrupt conduction passing onto the back wall through fibers deep to the roof line lesions. Passing around the margins of the right veins the septal line can be completed after identification and avoidance of the phrenic nerve course

Upon completion of the PWI, if there is persisting connection, the lasso is placed in the veins to determine earliest activation. Once earliest vein is identified, circular or other multipolar mapping catheter signals within the vein are used to determine if break through is anterior or posterior. With these signals as a reference, the earliest breakthrough is identified with the mapping catheter for targeted ablation to achieve PWI. This may be done in sinus rhythm or atrial fibrithation if activation within the ring is sufficiently organized. PWI is confirmed with demonstration of exit and entrance block in SR and pacing from coronary sinus.

The advantage of this technique is that it limits ablation over the posterior wall and potentially reduces risk of atrio-esophageal fistula (AEF). Ablation and fluoroscopy times may be comparable to other techniques.

Disadvantages. With a single ablation line, a single reconnection leads to reconnection in both set of pulmonary veins and posterior wall. Liner ablation over the roof and inferior LA may lead to reentry causing left atrial flutter. This approach is associated with longer procedure, RF and fluoroscopy times compared with box isolation ${ }^{33}$.

PWI approach 3:

\section{PVI with very wide posterior antral lines adjoining at the mid posterior wall}

This approach is a modified PVI with the posterior lines extending to the mid portion of the posterior left atrium (figure 3). After completing ablation over the anterior aspect of the pulmonary veins, ablation is extended from the superior aspect of the pulmonary veins towards the midpoint on the posterior wall between the superior pulmonary veins (SPV). A single vertical linear ablation is then performed over the mid posterior wall down to the 
midpoint of the inferior pulmonary veins (IPV). The ablation then continues towards the inferior most aspect of the respective veins and connects to the anterior ablation points to complete antral PY isolation.

Advantage: A single mid posterior line is shared by both the left and right circumferential lesion set with the potential to reduce ablation time on the posterior wall. This may be the preferred lesion set when the esophagus lies closer to the PVs adjacent to the usual posterior antral line performed by the operator.

Disadvantages: This approach isolates a smaller area of the posterior wall compared with the other approaches. Unlike the other techniques, there is little published on the success and safety of this approach.

PWI approach 4:

\section{Posterior wall ablation: debulking}

This refers to the approach pioneered by Natale and co-workers. Upon completion of the pulmonary vein isolation, the LASSO is placed on the posterior wall. The earliest electrograms are targeted with focal ablations until there is no electrical activity on the posterior wall (figure 2). This technique is performed in combination with further extensive ablation involving coronary sinus and the septal aspects of the right pulmonary veins.

Advantages: This technique does not rely on achieving block through linear ablation, but rather extensive local ablation.

Disadvantages: This approach is associated with longer procedure, RF and fluoroscopy times compared with box isolation ${ }^{18}$. Although there may be theoretical concerns regarding greater risk of oesophageal injury this has not been published to date.

\section{Achieving durable posterior LA isolation}

Posterior wall isolation is confirmed using the circular mapping catheter for the presence of electrical silence or spontaneous posterior wall potentials (PWPs) (figure 5). PWI is confirmed through the identification of entrance and exit block. Exit block is confirmed utilizing the ablator to pace the PW in the absence of dissociated PWPs. Adenosine is administered to assess for dormant conduction $^{6}$. Boluses of intravenous adenosine $(12-18 \mathrm{mg})$ is administered through a large bore (preferably central) intravenous access immediately followed by a $20-30 \mathrm{ml} \mathrm{N} /$ saline push to assess for acute reconnection of the pulmonary veins and the posterior wall ${ }^{6,34,35}$. At least one nonconducted $P$ wave or a 3 -second pause needs to be documented as adequate adenosine response ${ }^{29}$. Any transient or persistent electrical reconnection in response to adenosine is followed by ablation at the site of earliest activity on the original lesion set and adenosine repeated until reconnection is no longer present. Finally, a 30-minute waiting period is employed from the time of successful isolation. 


\section{DISCUSSION:}

Atrio-esophageal fistula (AEF) is a serious risk associated with any ablation involving the posterior wall. The risk is present with standard PV circumferential ablation with no evidence to suggest that posterior wall isolation increases this risk. In accordance with the 2017 HRS guidelines, an esophageal temperature probe is recommended, although recent evidence suggests that it may lead to increased reconnection at sites of increased temp recordings ${ }^{36}$.

There is a risk of atypical flutters after PVI and this is the case with PWI. To date, there have not been randomized studies to compare the incidence of peri mitral flutters between the two approaches. The incidence of peri-mitral flutter with an approach that includes PVI plus posterior LA isolation is $3.7 \%-5.8 \%{ }^{10,37}$. However, a recent meta-analysis did not show any significant difference in the occurrence of flutter after PWI compared with PVI alone (RR $1.16 \mathrm{P}=0.034)^{15}$

To date, multicenter randomized studies comparing PVI vs PVI plus posterior wall isolation are yet to be completed. Nor are there randomized comparisons of the various strategies to achieve posterior wall isolation. As such, it is at the discretion of the operator as to whether this is incorporated into the AF ablation lesion set with the strategy determined by procedural expertise and patient /anatomic factors.

\section{CONCLUSION}

Posterior wall isolation is a common adjunct to standard PVI for catheter ablation for atrial fibrillation. There are a variety of techniques that may be employed depending on operator and patient considerations. Randomized studies are underway to determine the role of posterior wall isolation in catheter ablation for atrial fibrillation.

\section{REFERENCES}

1. Kirchhof $P$, Benussi S, Kotecha D, Ahlsson A, Atar D, Casadei B, Castella M, Diener HC, Heidbuchel H, Hendriks J, Hindricks G, Manolis AS, Oldgren J, Popescu BA, Schotten U, Van Putte B and Vardas R.2016 ESC Guidelines for the management of atrial fibrillation developed in collaboration with EACTS: The Task Force for the management of atrial fibrillation of the European Society of Cardiology (ESC)Developed with the special contribution of the European Heart Rhythm Association (EHRA) of the ESCEndorsed by the European Stroke Organisation (ESO). Europace. 2016.

2. Verma A, Jiang C-y, Betts TR, Chen J, Deisenhofer I, Mantovan R, Macle L, Morillo CA, Haverkamp W, Weerasooriya R, Albenque J-P, Nardi S, Menardi E, Novak P and Sanders P. Approaches to Catheter Ablation for Persistent Atrial Fibrillation. New England Journal of Medicine. 2015;372:1812-1822. 
3. Nademanee K, McKenzie J, Kosar E, Schwab M, Sunsaneewitayakul B, Vasavakul T, Khunnawat $\mathrm{C}$ and Ngarmukos T. A new approach for catheter ablation of atrial fibrillation: mapping of the electrophysiologic substrate. J Am Coll Cardiol. 2004;43:2044-53.

4. Marrouche NF, Wilber D, Hindricks G, Jais P, Akoum N, Marchlinski F, Kholmovski E, Burgon N, Hu N, Mont L, Deneke T, Duytschaever M, Neumann T, Mansour M, Mahnkopf C, Herweg B, Daoud E, Wissner E, Bansmann $P$ and Brachmann J. Association of atrial tissue fibrosis identified by delayed enhancement MRI and atrial fibrillation catheter ablation: the DECAAF study. JAMA. 2014;311:498-506.

5. JaïS P, O'Neill MD, Takahashi Y, JÖNsson A, Hocini M, Sacher F, Sanders P, Kodali S, Rostock $T$, Rotter M, ClÉMenty J and HaïSsaguerre M. Stepwise Catheter Ablation of Chronic Atrial Fibrillation:Importance of Discrete Anatomic Sites for Termination. J Cardiovasc Electrophysiol. 2006;17:S28-S36.

6. MoLellan AJA, Prabhu S, Voskoboinik A, Wong MCG, Walters TE, Pathik B, Morris GM, Nisbet $A$, Lee $G$, Morton JB, Kalman JM and Kistler PM. Isolation of the posterior left atrium for patients with persistent atrial fibrillation: routine adenosine challenge for dormant posterior left atrial conduction improves long-term outcome. Europace. 2017.

7. Douglas $\mathrm{YL}$, Jongbloed $\mathrm{MR}$, Gittenberger-de Groot AC, Evers D, Dion RA, Voigt $\mathrm{P}$, Bartelings MM, Schalij MJ, Ebels T and DeRuiter MC. Histology of vascular myocardial wall of left atrial body after pulmonary venous incorporation. Am J Cardiol. 2006;97:662-70.

8. Douglas YL, Jongbloed MR, Deruiter MC and Gittenberger-de Groot AC. Normal and abnormal development of pulmonary veins: state of the art and correlation with clinical entities. Int J Cardiol. 2011;147:13-24.

9. Calkins H, Ho SY, Angel Cabrera J, Della Bella P, Farre J, Kautzner J and Tchou P. Anatomy of the Left Atrium and Pulmonary Veins Atrial Fibrillation Ablation: Blackwell Publishing Ltd; 2008: 1-10.

10. Sanders R, Hocini M, Jaïs P, Sacher F, Hsu L-F, Takahashi Y, Rotter M, Rostock T, Nalliah CJ, Clémenty J and Haissaguerre M. Complete isolation of the pulmonary veins and posterior left atrium in chronic atrial fibrillation. Long-term clinical outcome†. European Heart Journal. 2007;28:18621871.

11. HoSY, Sanchez-Quintana D, Cabrera JA and Anderson RH. Anatomy of the left atrium: implications for radiofrequency ablation of atrial fibrillation. J Cardiovasc Electrophysiol. 1999;10:1525-33.

12. Markides $V$, Schilling RJ, Ho SY, Chow AW, Davies DW and Peters NS. Characterization of left atrial activation in the intact human heart. Circulation. 2003;107:733-9. 
13. Platonov PG, Ivanov V, Ho SY and Mitrofanova L. Left atrial posterior wall thickness in patients with and without atrial fibrillation: data from 298 consecutive autopsies. J Cardiovasc Electrophysiol. 2008;19:689-92.

14. Kumar P, Bamimore AM, Schwartz JD, Chung EH, Gehi AK, Kiser AC, Hummel JP and Mounsey JP. Challenges and Dutcomes of Posterior Wall Isolation for Ablation of Atrial Fibrillation. J Am Heart Assoc. 2016;5.

15. He X, Zhou $Y$, Chen $Y$, Wu L, Huang $Y$ and He J. Left atrial posterior wall isolation reduces the recurrence-of atrial fibrillation: a meta-analysis. J Interv Card Electrophysiol. 2016.

16. Kim JS, Shin SY, Na JO, Choi CU, Kim SH, Kim JW, Kim EJ, Rha SW, Park CG, Seo HS, Oh DJ, Hwang $\mathrm{C}$ and Lim $\mathrm{HE}$. Does isolation of the left atrial posterior wall improve clinical outcomes after radiofrequency catheter ablation for persistent atrial fibrillation?: A prospective randomized clinical trial. Int J Cardiol:2015;181:277-83.

17. O'Neill L, Hensey M, Nolan W and Keane D. Clinical outcome when left atrial posterior wall box isolation is included as a catheter ablation strategy in patients with persistent atrial fibrillation. $J$ Interv Card Electrophysiol. 2015;44:63-70.

18. BairR, Di Biase L, Mohanty P, Trivedi C, Dello Russo A, Themistoclakis S, Casella M, Santarelli P, Fassini G, Santangeli P, Mohanty S, Rossillo A, Pelargonio G, Horton R, Sanchez J, Gallinghouse J, Burkhardt JD, Ma CS, Tondo $\mathrm{C}$ and Natale A. Proven isolation of the pulmonary vein antrum with or without left atrial posterior wall isolation in patients with persistent atrial fibrillation. Heart Rhythm. 2016;13:132-40.

19. Wyn GJ, Das M, Bonnett L, Panikker S, Wong T and Gupta D. Efficacy of Catheter Ablation for Persistent Atrial Fibrillation: A Systematic Review and Meta-Analysis of Evidence From Randomized and Nonrandomized Controlled Trials. Circ. 2014;7:841-852.

20. Mohanty S, Gianni C, Mohanty P, Halbfass P, Metz T, Trivedi C, Deneke T, Tomassoni G, Bai R, Al-Ahmad A, Bailey S, Burkhardt JD, Gallinghouse GJ, Horton R, Hranitzky PM, Sanchez JE, Di Biase $L$ and Natale A. Impact of Rotor Ablation in Non-Paroxysmal AF Patients: Results from a Randomized Trial (OASIS). J Am Coll Cardiol. 2016.

21. Prabhu S, Taylor AJ, Costello BT, Kaye DM, McLellan AA, Voskoboinik A, Sugumar $H$, Lockwood SM, Stokes MB, Pathik B, Nalliah CJ, Wong GR, Azzopardi SM, Gutman S, Lee G, Layland J, Mariani JA, Ling L-h, Kalman JM and Kistler PM. Catheter Ablation Versus Medical Rate control in Atrial Fibrillation and Systolic Dysfunction (CAMERA-MRI). Journal of the American College of Cardiology 2017.

22. Cox JL, Schuessler RB, Lappas DG and Boineau JP. An 8 1/2-year clinical experience with surgery for atrial fibrillation. Ann Surg. 1996;224:267-73; discussion 273-5. 
23. Thomas SP, Lim TW, McCall R, Seow SC and Ross DL. Electrical isolation of the posterior left atrial wall and pulmonary veins for atrial fibrillation: feasibility of and rationale for a single-ring approach. Heart Rhythm. 2007;4:722-30.

24. Kumagai-K, Muraoka S, Mitsutake C, Takashima H and Nakashima H. A new approach for complete isolation of the posterior left atrium including pulmonary veins for atrial fibrillation. $J$ CardiovascElectrophysiol. 2007;18:1047-52.

25. Ernst S, Ouyang F, Schneider B and Kuck KH. Prevention of atrial fibrillation by complete compartmentalization of the left atrium using a catheter technique. J Cardiovasc Electrophysiol. 2000;11:686-90.

26. Pump A, Di Biase L, Price J, Mohanty P, Bai R, Santangeli P, Mohanty S, Trivedi C, Yan RX, Horton R, Sanchez JE, Zagrodzky J, Bailey S, Gallinghouse GJ, Burkhardt JD and Natale A. Efficacy of catheter ablation in nonparoxysmal atrial fibrillation patients with severe enlarged left atrium and its impact on left atrial structural remodeling. J Cardiovasc Electrophysiol. 2013;24:1224-31.

27. Kistler PM, Ho SY, Rajappan K, Morper M, Harris S, Abrams D, Sporton SC and Schilling RJ. Electrophysiologic and anatomic characterization of sites resistant to electrical isolation during circumferential pulmonary vein ablation for atrial fibrillation: a prospective study. J Cardiovasc Electrophysiol. 2007;18:1282-8.

28. Sy RW, Gula LJ, Leong-Sit P, Klein GJ, Yee R, Krahn AD, Bentley C, Razak S and Skanes AC. Complete antral encirclement is not required for pulmonary vein isolation. Heart Rhythm. 2011;8:1622.

29. Calkins H, Hindricks G, Cappato R, Kim YH, Saad EB, Aguinaga L, Akar JG, Badhwar V, Brugada J, Camm J, Chen PS, Chen SA, Chung MK, Nielsen JC, Curtis AB, Wyn Davies D, Day JD, d'Avila A, de Groot N, Di Biase L, Duytschaever M, Edgerton JR, Ellenbogen KA, Ellinor PT, Ernst S, Fenelon G, Gerstenfeld EP, Haines DE, Haissaguerre M, Helm RH, Hylek E, Jackman WM, Jalife J, Kalman JM, Kautzner J, Kottkamp H, Kuck KH, Kumagai K, Lee R, Lewalter T, Lindsay BD, Macle L, Mansour M, Marchlinski FE, Michaud GF, Nakagawa H, Natale A, Nattel S, Okumura K, Packer D, Pokushalov E, Reynolds MR, Sanders P, Scanavacca M, Schilling R, Tondo C, Tsao HM, Verma A, Wilber DJ and Yamane T. 2017 HRS/EHRA/ECAS/APHRS/SOLAECE expert consensus statement on catheter and surgical ablation of atrial fibrillation: executive summary. J Interv Card Electrophysiol. 2017.

30. Kochhauser S, Jiang CY, Betts TR, Chen J, Deisenhofer I, Mantovan R, Macle L, Morillo CA, Haverkamp W, Weerasooriya R, Albenque JP, Nardi S, Menardi E, Novak P, Sanders P, Verma A and Investigators SAI. Impact of acute atrial fibrillation termination and prolongation of atrial fibrillation cycle length on the outcome of ablation of persistent atrial fibrillation: A substudy of the STAR AF II trial. Heart Rhythm. 2017;14:476-483.

31. YuHT, Shim J, Park J, Kim I-S, Kim T-H, Uhm J-S, Joung B, Lee M-H, Kim Y-H and Pak H-N. Pulmonary Vein Isolation Alone Versus Additional Linear Ablation in Patients With Persistent Atrial 
Fibrillation Converted to Paroxysmal Type With Antiarrhythmic Drug Therapy: A Multicenter, Prospective, Randomized Study Circ Arrhythm Electrophysiol. 2017;10.

32. Lim TW, Koay CH, McCall R, See VA, Ross DL and Thomas SP. Atrial arrhythmias after singlering isolation of the posterior left atrium and pulmonary veins for atrial fibrillation: mechanisms and management. Circ Arrhythm Electrophysiol. 2008;1:120-6.

33. Lim TW, Koay CH, See VA, McCall R, Chik W, Zecchin R, Byth K, Seow SC, Thomas L, Ross DL and Thomas SP. Single-ring posterior left atrial (box) isolation results in a different mode of recurrence-compared with wide antral pulmonary vein isolation on long-term follow-up: longer atrial fibrillation-free survival time but similar survival time free of any atrial arrhythmia. Circ Arrhythm Electrophysiol. 2012;5:968-77.

34. Prabhu S, Mackin V, McLellan AJ, Phan T, McGlade D, Ling LH, Peck KY, Voskoboinik A, Pathik B, Nalliah C,,Wong GR, Azzopardi SM, Lee G, Mariani J, Taylor AJ, Kalman JM and Kistler PM. Determining the Optimal Dose of Adenosine for Unmasking Dormant Pulmonary Vein Conduction Following Atrial Fibrillation Ablation: Electrophysiological and Hemodynamic Assessment. DORMANT-AF Study. J Cardiovasc Electrophysiol. 2017;28:13-22.

35. Macle L, Khairy P, Weerasooriya R, Novak P, Verma A, Willems S, Arentz T, Deisenhofer I, Veenhuyzen G, Scavee C, Jais P, Puererfellner H, Levesque S, Andrade JG, Rivard L, Guerra PG, Dubuc $M$, Thibault $B$, Talajic $M$, Roy $D$, Nattel $S$ and investigators At. Adenosine-guided pulmonary vein isolation for the treatment of paroxysmal atrial fibrillation: an international, multicentre, randomised superiority trial. Lancet. 2015;386:672-9.

36. Tran VN, Kusa S, Smietana J, Tsai WC, Bhasin K, Teh A, Syros G, Singh A, Choudry S, Miller MA, KoruthJ, D'Avila A, Dukkipati SD and Reddy VY. The relationship between oesophageal heating during left atrial posterior wall ablation and the durability of pulmonary vein isolation. Europace. 2017.

37. Davies EJ, Lines I, Dalrymple Hay M and Haywood GA. The Late Electrophysiological Consequences Of Posterior Wall Isolation In Patients With Atrial Fibrillation. Journal of Atrial Fibrillation. 2015;8:1194.

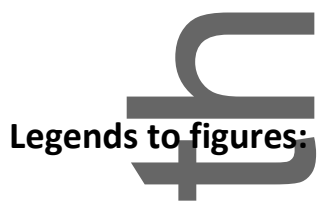

Figure 1: Posterior left atrium anatomy.

(A) Post mortem specimen of left atrium (LA) that has been everted. An abrupt change in fiber orientation occurs at the lateral margin (broken line) of the septopulmonary bundle (SPB). The figure illustrates the average thickness of the posterior wall ( $1 \mathrm{~cm}$ away from the veno-atrial junction) and LA roof. (Adapted from Markides et al Circulation 2003)

(B) CT reconstruction in the left lateral view with caudal tilt demonstrating the typical relationship 
between the esophagus and left atrium. The esophagus is in close proximity to the lower half of the posterior wall. The esophagus tends to separate off the posterior wall above the level of the mid posterior wall $(*)$.

$\mathrm{RS}=$ Right superior pulmonary vein. $\mathrm{RI}=$ Right inferior pulmonary vein. $\mathrm{LS}=$ Left superior pulmonary vein. $\mathrm{LI}=$ Left inferior pulmonary vein. $\mathrm{LAA}=$ Left atrial appendage.

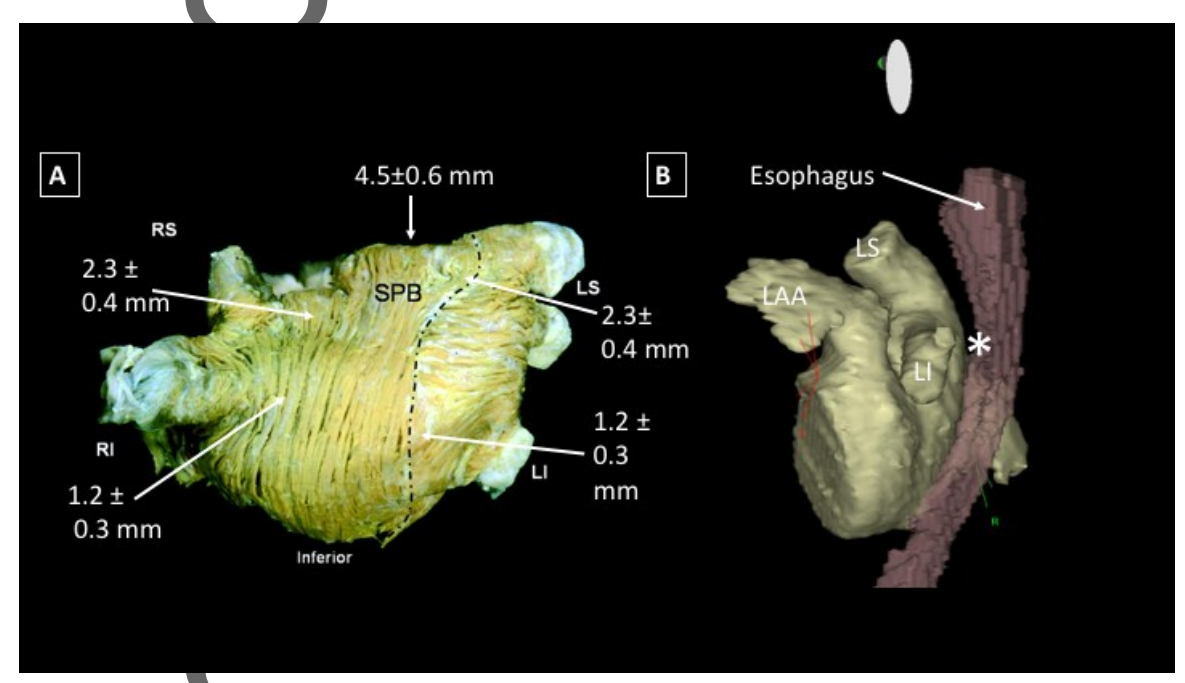

Figure 2: Posterior wall isolation techniques - PA view.

(A) Wide antral pulmonary vein isolation plus posterior box isolation. (B) Single ring approach. (C) Touching rings approach \& (D) Targeting earliest activation with debulking technique involving extensive posterior wall ablation.

$\mathrm{RS}=$ Right superior pulmonary vein. $\mathrm{RI}=$ Right inferior pulmonary vein. $\mathrm{LS}=$ Left superior pulmonary vein. $\mathrm{LI}=$ Left inferior pulmonary vein.

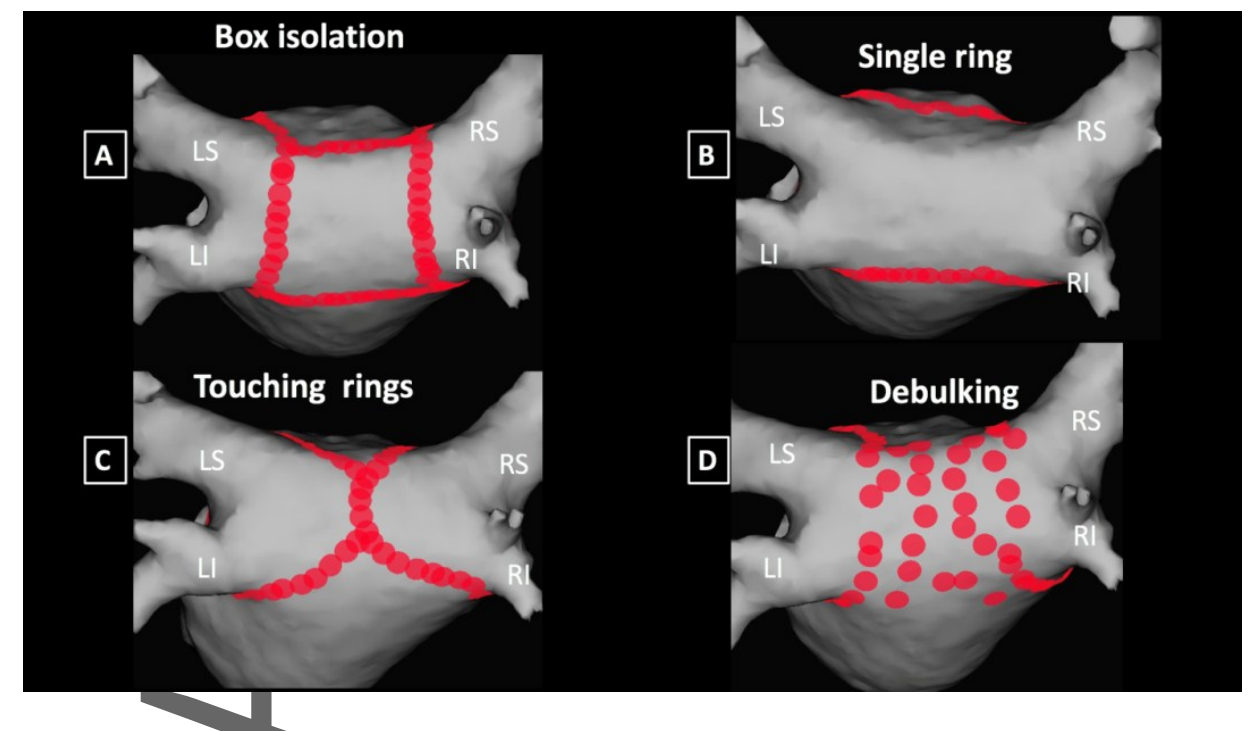

Figure 3: Wide antral pulmonary vein isolation plus posterior box isolation. Step 1: Wide antral circumferential ablation (WACA) isolating the right and left pulmonary veins. Step 2: Inferior / Floor 
line. Step 3: Roof line. $\mathrm{RS}=$ Right superior pulmonary vein. $\mathrm{RI}=$ Right inferior pulmonary vein. $\mathrm{LS}=$ Left superior pulmonary vein. $\mathrm{LI}=$ Left inferior pulmonary vein.

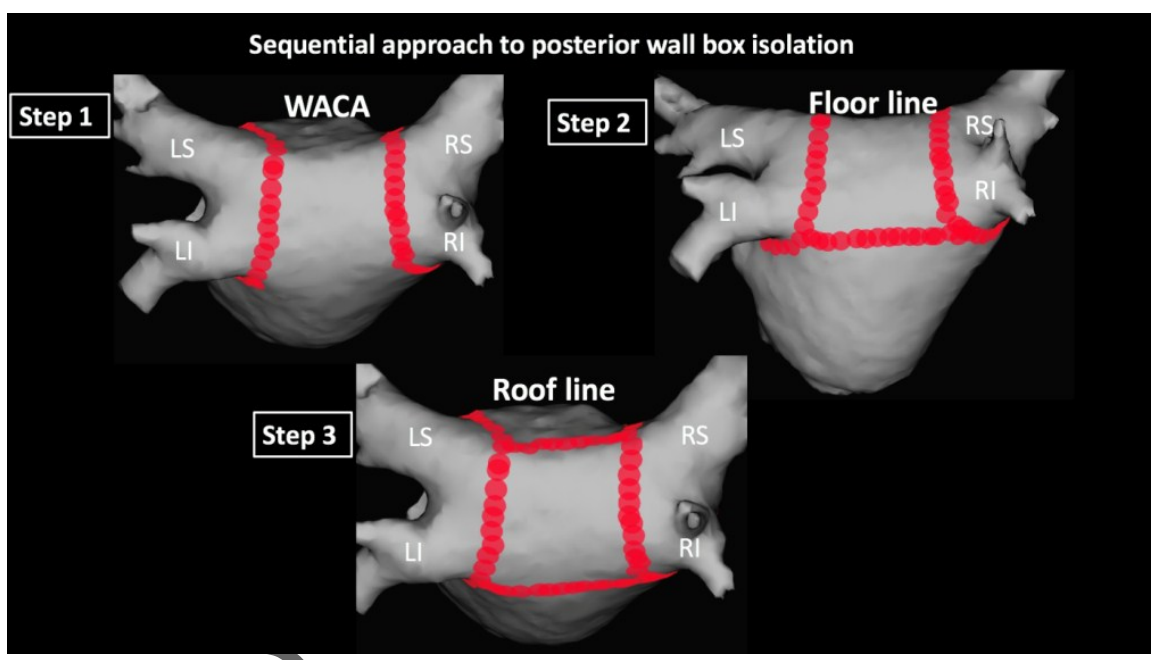

Figure 4. LA Floor line. (A) Demonstrating a CT image integration displaying PA view with left caudal tilt of the left atrium. The mapping catheter (blue), ablation catheter (grey with green tip) and completed CPVI around right and left-sided veins are seen. Ablation catheter is positioned near the LIPV at the start of the inferior line. (B) Position of ablation catheter to test for bidirectional block over the inferior line. (C) EGM demonstrating floor line block. Distal CS is being paced at $600 \mathrm{~ms}$ (100bpm) with short conduction time to posterior wall $(78 \mathrm{~ms})$ in the first beat with earliest activation on PV pole 9,10. The second and third beats demonstrate change in activation with earliest now at pole 17,18 with conduction time extended to $128 \mathrm{~ms}$ and wide spaced doubles on the distal bipole of the ablation catheter (ABLd).

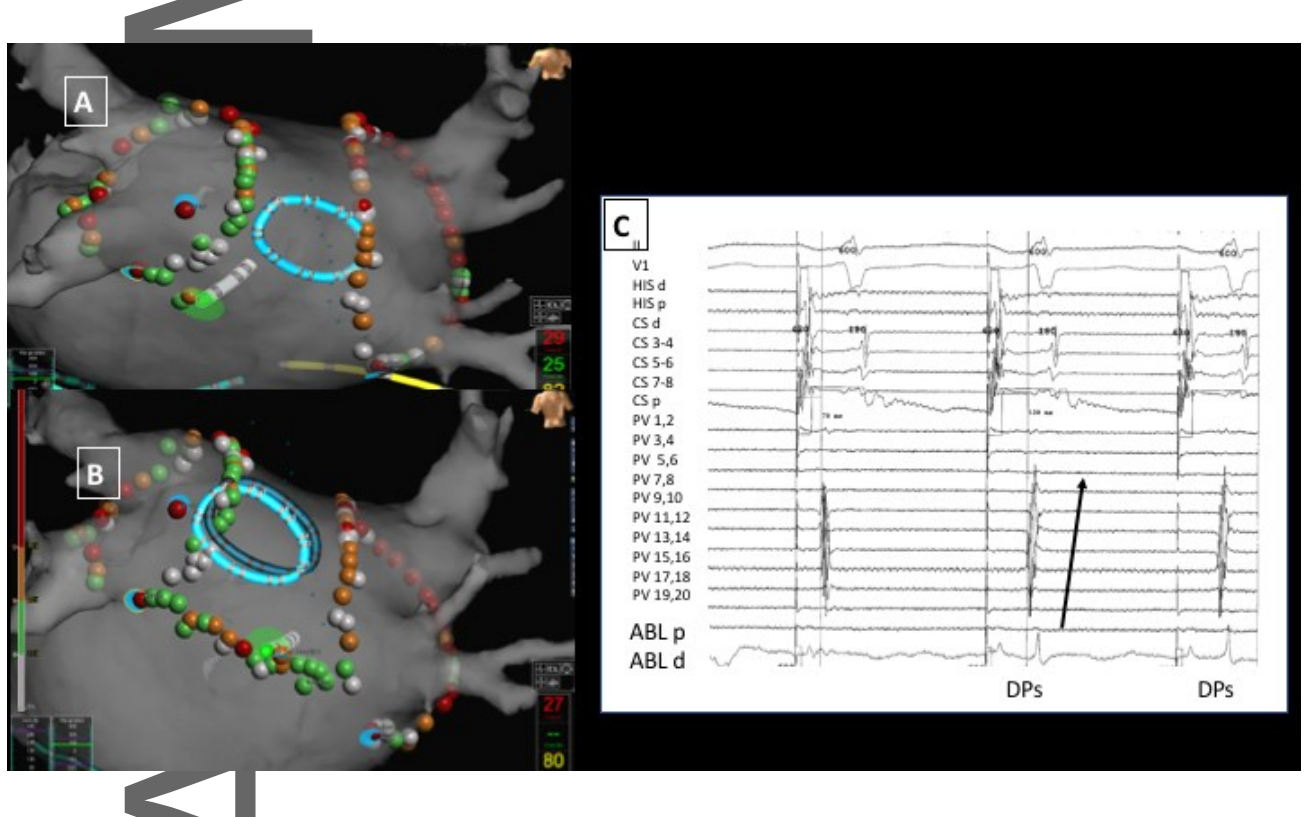

Figure 5: LA roofline.

This article is protected by copyright. All rights reserved. 
(A - C) Sequential fluoroscopic view (PA) of the cardiac chambers demonstrating catheter positions demonstrating RIFLE technique. Decapolar catheter in the coronary sinus. Quadripolar catheter in the HIS position. Mapping Lasso catheter in the posterior wall. The LSPV positioned is outlined with the white lines to demonstrate the relationship of the sheath and catheter to the LSPV. (A) Step 1: SL1 sheath and catheter advanced towards LSPV with the ablation catheter about 4-5 cm out of the sheath and D flexed pointing inferiorly. (B) Step 2: The sheath is then clocked 180degrees pointing the ablation catheter to the right- sided veins. (C) Step 3: D curve can be released to bring the catheter tip in contact with the local tissue near the RSPV. (D) Demonstrating a CT image integration displaying PA view with cranial tilt of the left atrium. The mapping catheter (blue), ablation catheter (grey with green (tip) and completed PVI around right- and left-sided veins are seen. Ablation catheter is positioned near the roof of RSPV with RIFLE technique. (E) Completion of the roof line.

$\mathrm{RSPV}=$ right superior pulmonary vein. $\mathrm{LSPV}=$ left superior pulmonary vein .

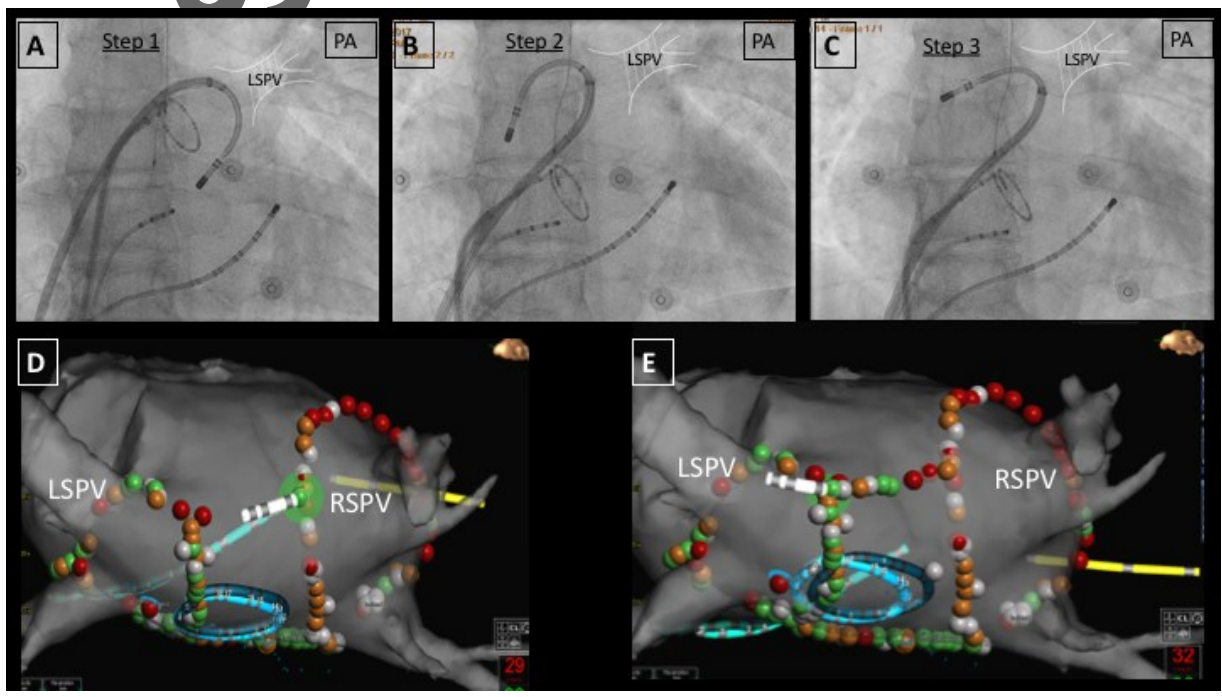

Figure 6: Posterior wall isolation. Spontaneous potential.

(A) PA view demonstrating completed roof and inferior line in relation to the CPVI lines. (B) Electrically isolated left atrial posterior wall with spontaneous posterior wall potential $\left({ }^{*}\right)$ and exit block.

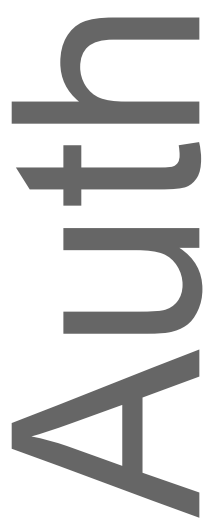

This article is protected by copyright. All rights reserved. 


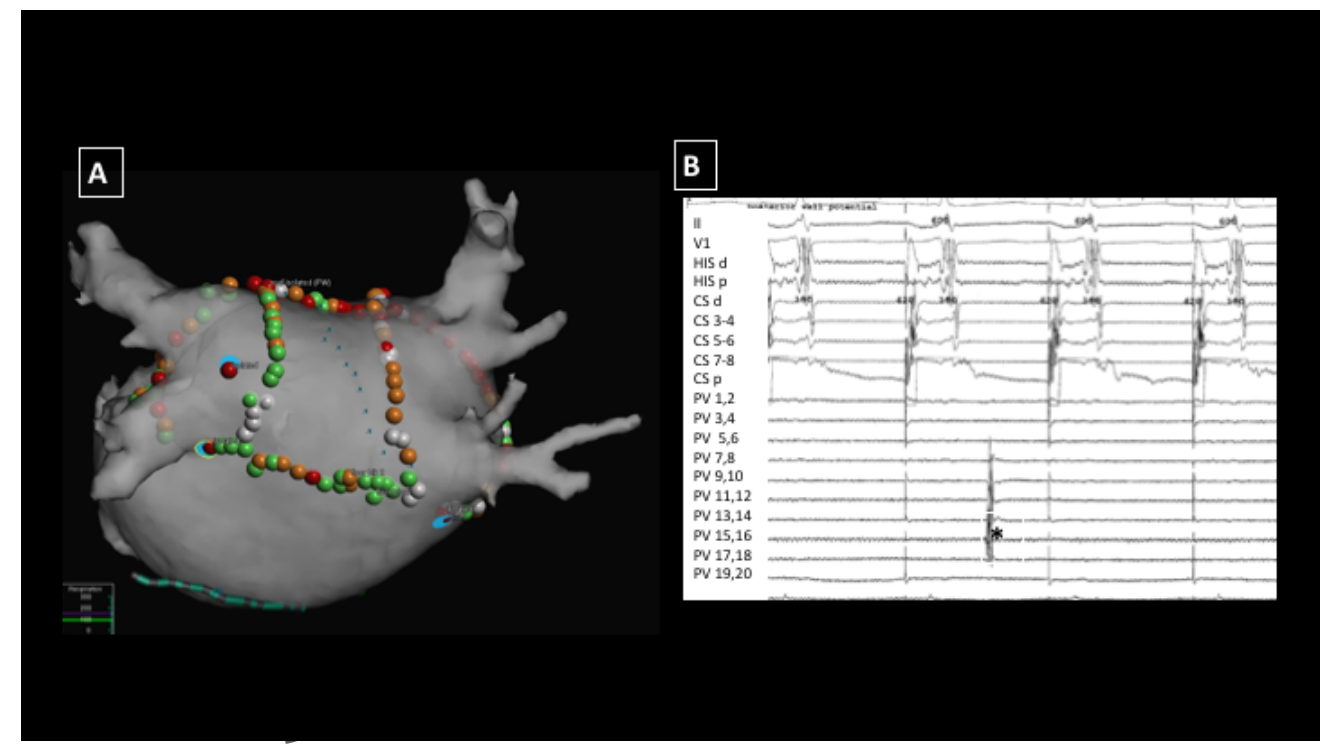

TABLES:

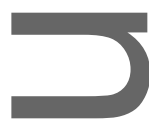

Table 1: RIFLE technique. Correlation between sheath and catheter tip movement.

\begin{tabular}{|l|l|}
\hline Sheath movement & Anticipated catheter tip movement \\
\hline Clocking the sheath & Moves anteriorly / cranially \\
\hline Anti-clocking the sheath & Moves posteriorly / caudally \\
\hline Advancing the sheath into LSPV & Moves towards from right antrum to mid roof \\
\hline Withdrawing sheath and ablator & Moves towards roof and right pulmonary vein \\
\hline Withdrawing catheter into sheath & Straightens the catheter and increases contact with roof \\
\hline
\end{tabular}

This article is protected by copyright. All rights reserved. 


\section{University Library}

\section{- M M N E R VA A gateway to Melbourne's research publications}

Minerva Access is the Institutional Repository of The University of Melbourne

Author/s:

Sugumar, H;Thomas, SP;Prabhu, S;Voskoboinik, A;Kistler, PM

Title:

How to perform posterior wall isolation in catheter ablation for atrial fibrillation

Date:

2018-02-01

Citation:

Sugumar, H., Thomas, S. P., Prabhu, S., Voskoboinik, A. \& Kistler, P. M. (2018). How to perform posterior wall isolation in catheter ablation for atrial fibrillation. JOURNAL OF CARDIOVASCULAR ELECTROPHYSIOLOGY, 29 (2), pp.345-352. https://doi.org/10.1111/ jce.13397.

Persistent Link:

http://hdl.handle.net/11343/294069 